\title{
Reduction of the suction pressure of a liquid ring vacuum pump with a supersonic gas ejector
}

\author{
Róbert Olšiak ${ }^{1, *}$, Zoltán Fuszko ${ }^{1}$, and Zoltán Csuka ${ }^{1}$ \\ ${ }^{1}$ Slovak University of Technology, Faculty of M.E., Institute of energy machinery, Námestie slobody \\ 17, 81231 Bratislava, Slovakia
}

\begin{abstract}
A supersonic gas ejector in conjunction with a liquid ring vacuum pump is used for creating and maintaining vacuum in a chamber for technological purposes. In this paper the authors submit an overview about the problematics of suction pressure reduction with a supersonic gas ejector used as a pre-stage of a liquid ring vacuum pump. This system has also the function of a cavitation protection due to the higher pressure present at the suction throat of the vacuum pump. A part of this paper is devoted to the governing equations used at the definition of the flow through an ejector. The experimental studies are then carried out in or own laboratory for verification purposes.
\end{abstract}

\section{Introduction}

The vacuum pumps are technological devices used to create and maintain vacuum for technological purposes. In practice the vacuum means the state of diluted gas, which absolute pressure is lower than the atmospheric. Liquid ring vacuum pumps can be used at extraction of condensated vapor and gas mixtures. In this case a part of the pumped mixture is condensating in form of vapor inside the vacuum pump, while the condensate is drained with the working fluid. At the other vacuum pump types this phenomenon is undesirable due to the present lubricating grease. The relatively low efficiency and the high minimal suction pressure can be considered as a disadvantage. This minimal pressure is limited by the used working fluid inside the vacuum pump. Is problematic to determine this correlation, because the parameters of the liquid ring vacuum pump are bounded not only with the type of used fluid (density, viscosity) but with the change of these properties in dependence on the temperature and pressure also.

The barrier of the minimal suction pressure, at which the vacuum pump can be used, is depending on the fluid properties, construction type, number of stages etc. This pressure is according to the following authors stated as: Bannwarth $5 \mathrm{kPa}$, Faragallah $15 \mathrm{kPa}$, Szabó 4$14 \mathrm{kPa}$. In the practice is a common requirement to reach a suction pressure, which is much lower than the listed values. This is not possible due to the occurrence of cavitation inside the vacuum pump. This can be partially solved by lowering the pressure ratio, so using a

\footnotetext{
* Corresponding author: robert.olsiak@stuba.sk
} 
multistage liquid ring vacuum pump, however even this solution is unacceptable from the viewpoint of long-term run. The suction pressure can be lowered using a gas ejector as a pre-stage of the vacuum pump. This arrangement can reduce the pressure up to $1 / 5$ of the initial pressure.

\section{The options of the suction pressure reduction}

The ejector as a technological device is due to the simple construction and operational reliability are widely used at different applications. It is basically a device, in which the primary medium entrains the secondary driven medium, accelerates it to supersonic speed at reduced pressure. The pressure measured at the ejector inlet $\mathrm{p}_{2}$ is decisive to achieve the design pressure in the considered pressure chamber. From the viewpoint of ejector design, there are some limiting parameters (Figure 1): pressure measured at the ejector outlet $\mathrm{p}_{3}$, mass flow of the mixture $m_{3}$, which are corresponding to the occurrence of cavitation inside the vacuum pump. The values of the primary (driving) fluid $\mathrm{p}_{1}$ and $\mathrm{m}_{1}$ are used at the design of the primary supersonic nozzle. In case that the ejector is correctly designed, it will extend the working range of the liquid ring vacuum pump as seen on the figure below.
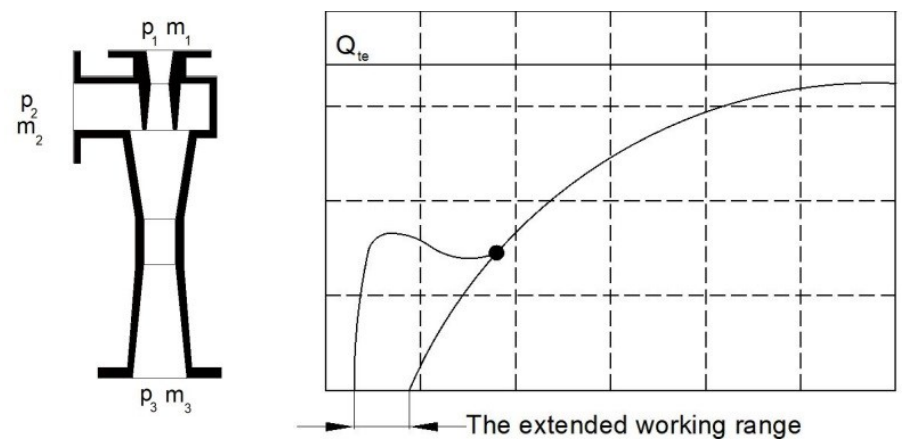

Fig. 1. The construction of a supersonic ejector (left) and the performance characteristics of a liquid ring vacuum pump with and without a supersonic ejector pre-stage.

From this simple analysis results that for the correct design of a supersonic ejector is necessary to know the following parameters:

- The required minimal suction pressure $\mathrm{p}_{2}$ at the chamber throat.

- The required mixture mass flow $\mathrm{m}_{2}$ at the chamber throat.

- The minimal suction pressure $\mathrm{p}_{3}$ of the vacuum source.

It is obvious that the pressure $\mathrm{p}_{2}$ and mass flow $\mathrm{m}_{2}$ are the chosen parameters of the designed ejector, the suction pressure $\mathrm{p}_{3}$ and mass flow $\mathrm{m}_{3}$ can be specified experimentally, which is the most commonly used method, or with theoretical calculation according to the geometrical dimensions of the device, operational data and properties of the working fluid.

\section{The governing equations}

The mostly used ejector design method is based on a 1-dimensional analysis of compressible gas flow. This method was first time proposed by Keenan (1942) and Neumann (1950) and it uses the following assumptions:

- The kinetic energy on the inlet of both fluids is negligible.

- The two working fluids are considered as ideal gases.

- At the end of the mixing chamber are both of the fluid uniformly mixed, without occurring shocks inside the mixing chamber. 
Based on these assumptions the equations describing the character of the flow from the inlets to the outlet can be given as follows [1]:

-the momentum equation

$$
p_{1} A_{1}+\rho_{1} Q_{1} v_{1}+p_{2} A_{2}+\rho_{2} Q_{2} v_{2}=p_{3} A_{3}+\rho_{3} Q_{3} v_{3}
$$

-the continuity equation

$$
\rho_{1} Q_{1}+\rho_{2} Q_{2}=\rho_{3} Q_{3}
$$

-the energy equation for adiabatic flow

$$
\dot{m}_{1}\left(h_{1}+\frac{v_{1}^{2}}{2}\right)+\dot{m}_{2}\left(h_{2}+\frac{v_{2}^{2}}{2}\right)=\dot{m}_{3}\left(h_{3}+\frac{v_{3}^{2}}{2}\right)
$$

The required compression ratio $(\mathrm{CR})$ of an ejector determines the ratio of the static pressures on the ejector exit (identic to the pressure measured at the inlet throat of the vacuum pump) to the pressure measured at the secondary suction throat of the ejector. The entrainment ratio (ER) [2] expresses the relationship between the desired mass flow rates through the secondary and primary inlets of the ejector.

$$
\begin{gathered}
C R=\frac{p_{3}}{p_{2}}, \quad E R=\frac{\dot{m}_{2}}{\dot{m}_{1}}=\frac{p_{2}}{p_{1}} \frac{A_{2}}{A_{1}}\left(\frac{T_{1}}{T_{2}}\right)^{\frac{1}{2}} \frac{f_{b}\left(\kappa_{2}, M_{2}\right)}{f_{a}\left(\kappa_{1}, M_{1}\right)} \\
\text { where: } f(\kappa, M)=\frac{\dot{m}}{p A}\left(R T_{0}\right)^{\frac{1}{2}}=M\left[\kappa\left(1+\frac{\kappa-1}{2} M^{2}\right)\right]^{\frac{1}{2}}
\end{gathered}
$$

\section{Design method}

The motion of the working fluid is given by the pressure difference between the atmospheric air at the primary inlet and the minimal suction pressure given by the vacuum pump. Due to the small molecular weight of the used working fluid (atmospheric air), the value of the kinetic energy of the primary fluid depends on the outlet speed of the supersonic nozzle. For this reason is the nozzle considered as the most important part of an ejector. The mostly used nozzle geometry due to his simple design is the conical, with constant wall angle $\left(12^{\circ}-18^{\circ}\right)$. Since the outlet velocity field has a radial component also, it is necessary to introduce a correction factor, which takes into account the ratio of the ideal momentum of the conical nozzle, with the axial component of the velocity.

Parabolic de Laval nozzles take into account the presence of Prandtl-Mayer expansion. The shape of the nozzle is obtained either by an empirical method, or by the method of characteristics (MoC) (Figure 2).

The Prandtl-Meyer formula (6) is used to determine the Mach number at a given point of the flow field followed by the Mach angle calculation (7).

$$
\begin{gathered}
v(M)=\sqrt{\frac{\kappa+1}{\kappa-1}} \arctan \sqrt{\frac{\kappa-1}{\kappa+1}\left(M^{2}-1\right)}-\arctan \sqrt{M^{2}-1} \\
\mu=\arcsin \frac{1}{M}
\end{gathered}
$$



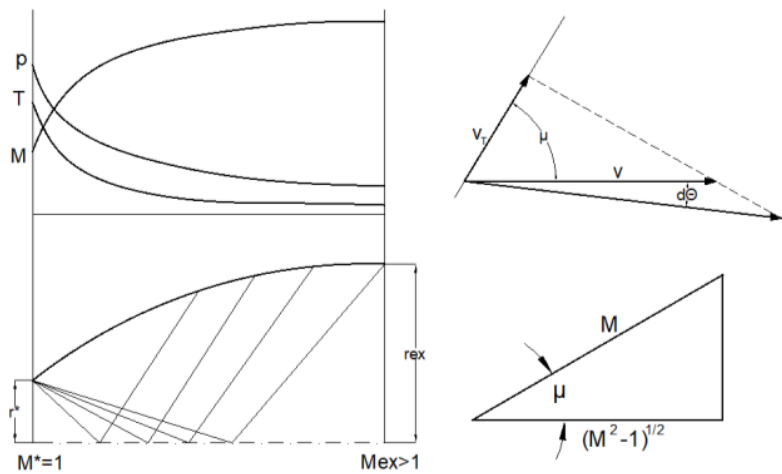

Fig. 2. The divergent portion of a parabolic de Laval nozzle with characteristic lines and distribution of the observed parameters (left), the velocity components with the angles used by the method of characteristics.

These equations alongside with the equations of quasi one-dimensional-isentropic flow of compressible fluids in ducts was an automated software developed [3]. The flowchart of the calculation can be seen on the following figure.

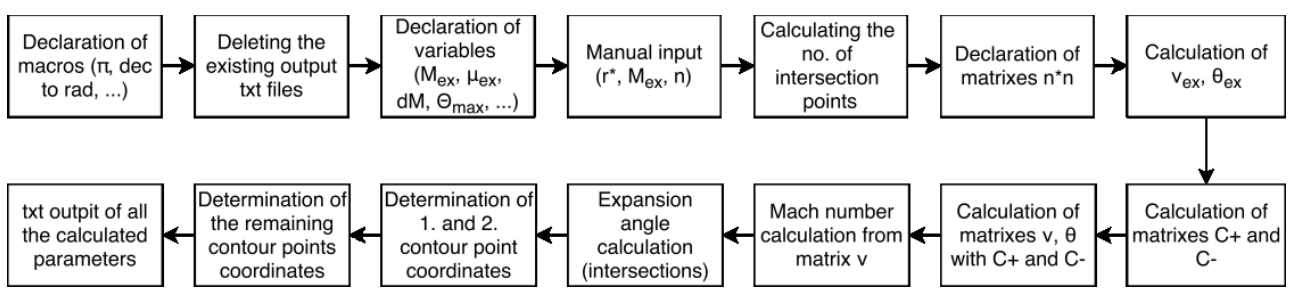

Fig. 3. Flowchart of the automated calculation process.

The high speed mixture of the primary air and secondary working fluid is entering the mixing chamber. The 1-D design model uses the constant-area mixing model, for which is the supersonic nozzle exit located directly on the cross section plane of the mixing chamber inlet (Figure 4).

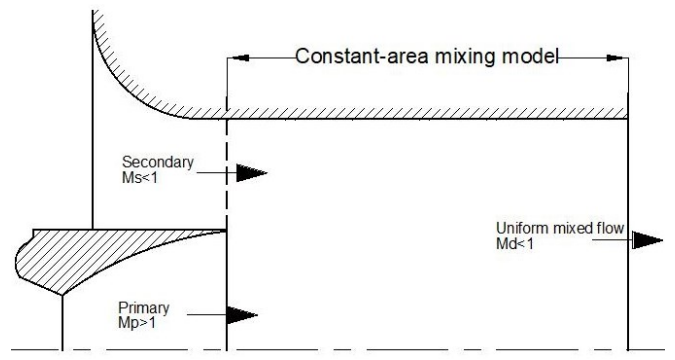

Fig. 4. Constant-area-mixing chamber.

With use of the ER alongside with equation (1) and the known flow rate at the diffuser outlet, the velocity at the exit of the mixing chamber can be obtained:

$$
v_{\text {mch,ex }}=\eta_{\text {mixing }}\left(\frac{\dot{m}_{1} v_{1}+\dot{m}_{2} v_{2}}{\dot{m}_{1}+\dot{m}_{2}}\right)
$$




\section{The experimental setup}

The experimental station consists of 2 main parts: a two-stage supersonic ejector and a liquid ring vacuum pump. The ejector stages are connected in series, so the outlet of the first stage is connected to the secondary suction of the second stage. The two-stage ejector is then added as a pre-stage to the vacuum pump as shown on the following figure.

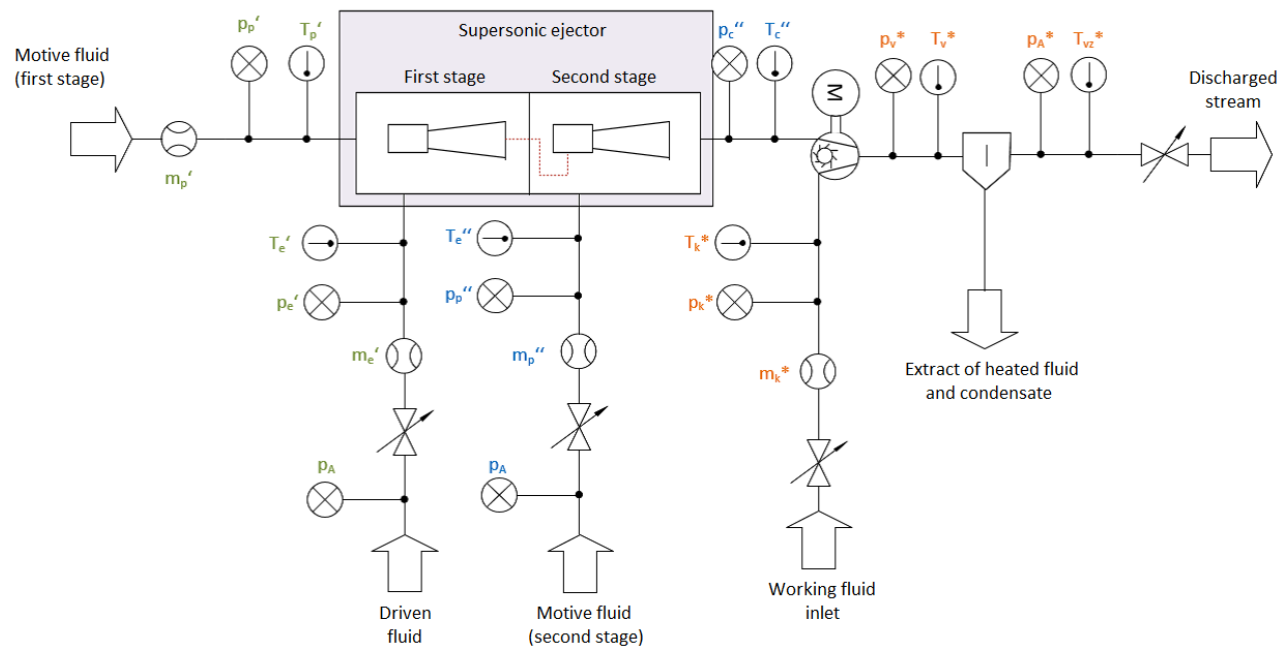

Fig. 5. The experimental setup (installed sensors and meters: m-flow rate, T-temperature, p-pressure).

The used liquid-ring vacuum pump type SZO is designed to extract air, chemically active and non-active vapors in metallurgical, chemical and food industry. The pump consists of a stationary housing in which is an eccentrically rotating impeller placed. The working liquid is fed through a separate circuit to the pump, which by the centrifugal force creates a cylindrical ring inside the casing which acts as a liquid piston. The gradually reducing volumes of the individual interblade chambers are compressing the pumped air, which reports to the discharge port of the pump. The working fluid is along with the pumped gas entering the separator, where the extraction of heated fluid and condensate occurs.

As the first step the digitization of the signals from the installed sensors was made [4]. Since the signal in the digital form has an electrical value, it was necessary to convert them to the values of the actual measured parameters. This step was done in the LABView environment with the known ranges of measuring sensors. The experiment has been made in 2 phases: at first was the vacuum pump itself measured, than the supersonic ejector was added to compare the characteristics of the both setups [5].

The experiment begins by running the pump of the external circuit to constantly deliver water to the pump. As the housing of the vacuum pump fills up to the desired level, the operating speed was set with the frequency converter. The shut-off valve located on the motive fluid inlet of both stages is fully opened. After the stabilization of the monitored values, the first point of the Q-p diagram was recorded. By gradually closing the valve on the secondary inlet port was the suction pressure reduced. At each stop was the actual point data recorded. The measured Q-p values were then imported to MS Excel, approximated, than the characteristics were plotted as shown on the following figure. 


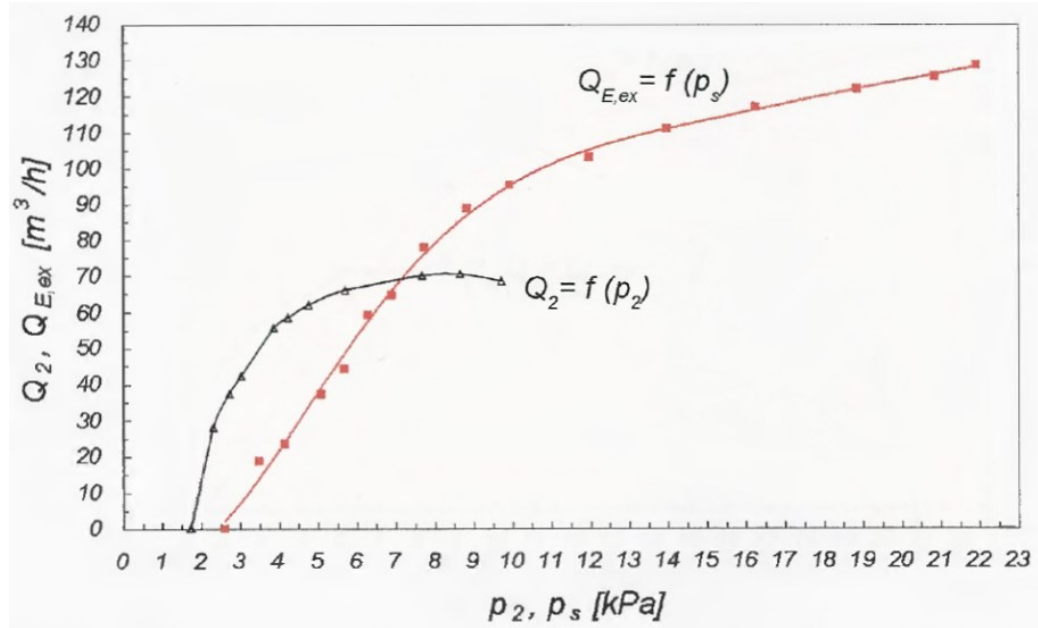

Fig. 6. Comparison of performance characteristics of the liquid ring vacuum pump with and without the supersonic ejector at 1400 RPM.

\section{Evaluation of the obtained experimental results}

At the analysis of the projected ejector performance characteristics should be remembered, that the performance is not given by the flow rate of air entering the suction throat of the vacuum pump. In case of an ejector used as a pre-stage, the flow rate of air measured at the secondary suction port (inlet no. 2 from Figure 1) can be considered as effective performance. This amount of air is exhausted from the vacuumed chamber. Also, the effective suction pressure is referred analogically to the secondary suction port. From the comparison of the performance characteristics is evident, that the added ejector had a positive impact on the performance of the whole vacuum system. At lower suction pressures (approx. under $10 \mathrm{kPa}$ abs.) is a significant performance increase present. The added ejector also extended the working range of the vacuum pump, so the vacuum system can be operated in a wider range of suction pressures in cavitation-free regime.

\section{References}

1. Ch. Liao, Gas ejector modeling (dissertation thesis, Texas, 2008)

2. P. Vician, M. Palacka, M. Holubčík, Effect of ext. temp. on performance of bin dryer, AIP Conference Proceedings, 1889, 020064 (2016)

3. M. Puškár, T. Brestovič, N. Jasminská, Num. sim. and exp. analysis of acoustic wave influences on brake mean eff. pressure in thrust-ejector inlet pipe of combustion engine, Int. Journal of Vehicle Design, 67, 63-76 (2015)

4. J. Jablonská, M. Mahdal, M. Kozubková, Spectral an. of pressure, noise and vibration velocity measurement in cavitation, The Journal of Institute of Measurement Science of Slovak Academy of Sciences, 17, 250-256 (2017)

5. S. Daneshmand, C. Aghanajafi, A. Bahrami, Analytical and experimental methods of design for supersonic two-stage ejectors, Int. Journal of Aerospace and Mech. Eng., 3, $2(2009)$ 